\title{
Optimal Design for a NEO Tracking Spacecraft Formation
}

\author{
Christie Alisa Maddock and Massimiliano Vasile
}

\begin{abstract}
The following paper presents the design and methodology for developing an optimal set of spacecraft orbits for a NEO tracking mission. The spacecraft is designed to fly in close formation with the asteroid, avoiding the nonlinear gravity field produced by the asteroid. A periodic orbit is developed, and the initial conditions are optimized by use of a global optimizer for constrained nonlinear problems. The asteroid Apophis (NEO 2004 MN4) was used as the case study due the potential impact with Earth in 2036, and the need for more accurate ephemerides.
\end{abstract}

\section{INTRODUCTION}

$\mathrm{T}$ HE existence of asteroids and comets are nothing new to science. As the planets formed and their orbits began to stabilize, the collision rate among the drifting asteroids decreased dramatically, however has in no way stopped. As the population and occupied surface of the Earth increases, the impact of even a relatively small asteroid could be devastating. An asteroid spanning $200 \mathrm{~m}$ in diameter, for example, can produce a crater $4 \mathrm{~km}$ in size, assuming it only hits land and not water. The larger classes of asteroids, those over $1 \mathrm{~km}$ in diameter, are considered to be a global threat [1]. While various methods of asteroid deviation are being investigated, it is equally, if not more important to be able to identify and track, with a high degree of accuracy, the numerous Near Earth Objects (NEO) that exist in our solar system [2], [3].

In tracking an object, it is important to have an orbit that is in relative proximity to the asteroid at all times. On-board measuring systems, such as laser and radar tracking instruments determine the distance between the spacecraft and a particular spot on the surface of the asteroid. In the case of multiple spacecraft, and/or multiple readings by the same spacecraft over time, triangulation techniques can be easily applied to determine the ephemerides of the asteroid with a high degree of accuracy.

This paper presents the design and optimization of a set of orbits to allow a spacecraft to fly in formation with Apophis, orbiting around the Sun. The asteroid Apophis was chosen as the test case based on the popularity of this particular asteroid in the field today due to two possible collisions with Earth in 2036. While the cumulative probability of impact is small, only $2.2 \mathrm{e}-5$ according the NASA Near Earth Object

Manuscript received March 15, 2007.

C. Maddock is Ph.D. student with the Space Advanced Research Team in the Department of Aerospace Engineering, University of Glasgow, Glasgow, UK G12 8QQ (phone: +44 141330 8470; fax: +44 141330 8470; e-mail: c.maddock@aero.gla.ac.uk).

M. Vasile is a lecturer with the Space Advanced Research Team in the Department of Aerospace Engineering, University of Glasgow, Glasgow, UK G12 8QQ (e-mail: m.vasile@aero.gla.ac.uk).
Program [4], it highlights the lack of both quality and quantity of tracking measurements available. With the majority of data gained from Earth-based observation, there is a growing need for high accuracy, longer term measurements from a space-based platform.

Ideally the spacecraft should fly in formation with the asteroid at an almost constant distance from it. Finding the optimal set of initial conditions that provide a periodic motion with constant distance is can be formulated as a constrained global optimization problem.

A black box containing a model of the problem under investigation was programmed for the orbit of a single spacecraft flying in formation with the NEO. A hybrid deterministic-stochastic approach based on behaviorism was then used to characterize the search space and to find the desired set of initial conditions.

\section{Simulation Models}

The following section describes the dynamical models used in simulating the orbit of a formation of spacecraft about a NEO, in this case Apophis.

\section{A. Asteroid Model}

In the case of Apophis, current analysis data is based on 7 radar delay and Doppler measurements, and 731 optical observations over a period of 2.4 years [4]. Table 1 and Fig. 1 give the physical data and orbit diagram respectively. The physical dimensions are calculated assuming an ellipsoid model for the asteroid based on the mass and rotational velocity where $r_{1} \geq r_{2} \geq r_{3}$.

TABLE 1

PHYSICAL DATA OF APOPHIS 99942

\begin{tabular}{ccccc}
\hline \hline $\begin{array}{c}\text { Mass } \\
(\mathrm{kg})\end{array}$ & $\mathrm{r}_{1}$ & $\mathrm{r}_{2}$ & $\mathrm{r}_{3}$ & $\begin{array}{c}\text { Rotational } \\
\text { velocity }(\mu \mathrm{rad} / \mathrm{s})\end{array}$ \\
\hline $4.6 \mathrm{E}+10$ & 226.3 & 160.0 & 113.1 & 5.7 \\
\hline \hline
\end{tabular}

In a related study on a NEO deviation strategy [5], an analysis of the dynamical environment of Apophis was conducted in order to determine the feasibility of having the spacecraft orbit the asteroid using only it's gravitation field. The model also compared the effects of third-body perturbations from the Sun and Apophis on the spacecraft.

It was found that the perturbations near Apophis are either highly chaotic, or too weak relative to the solar effects to allow a spacecraft to easily orbit the asteroid. 


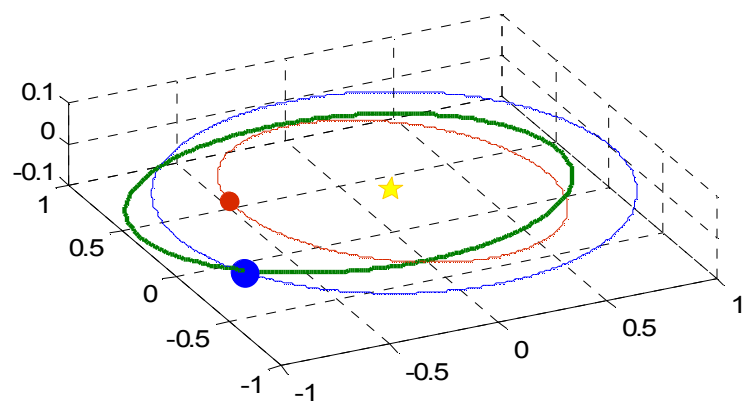

Fig. 1. Orbits of Earth (blue/thin), Venus (red/thin) and Apophis (bolded/green), showing the physical locations of each on the date 13 April 2036 08:53 GMT (one of the possible impact dates). All units are measured in standard astronomical units (AU) using the heliocentric Earth-equatorial reference frame.

An alternative approach is to fly the spacecraft in close formation with Apophis; as such, the spacecraft orbits around the Sun with the asteroid, but is not affected by its presence. In order to avoid unwanted perturbations caused by the asteroid, the minimum distance was set equal to the sphere of influence (SOI) of the gravity field of the asteroid. In a two-body problem, the sphere of influence occurs at the point where the gravitation field of the first body, e.g. the Sun, is equal to that of the second body, e.g. Apophis. Outside this area, the dominant gravitation force is the Sun.

\section{B. Equations of Motion}

The formation orbit can be thought of as a small orbit orbiting within a larger one. The smaller orbit orbits relative to a reference point, which in turn orbits around the Sun (see Fig. 2).

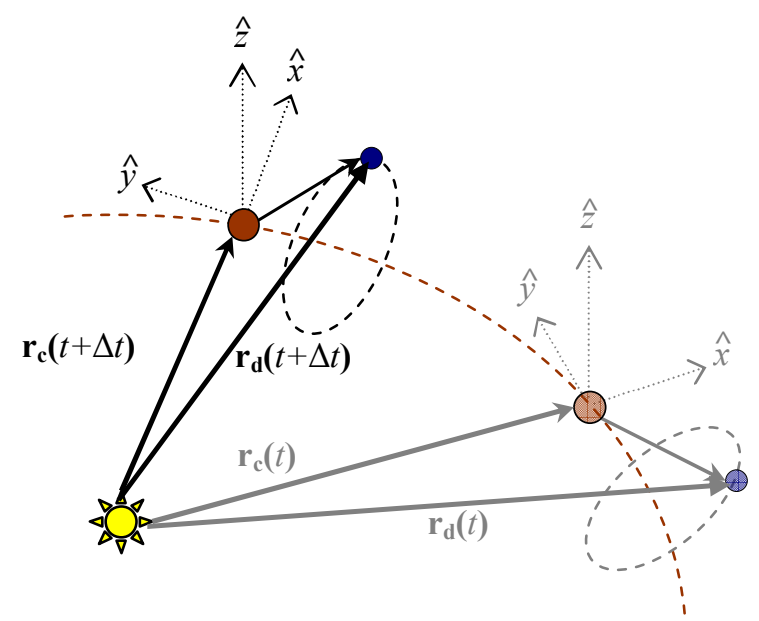

Fig. 2. Illustration of a chief orbit and a relative deputy spacecraft orbit flying in formation at two instants in time, $t$ and $(t+\Delta t)$. The vector from the sun to the chief spacecraft in inertial coordinates is given by $\mathbf{r}_{\mathfrak{c}}$, and from the sun to the formation, or deputy, spacecraft by $\mathbf{r}_{\mathbf{d}}$.

This type of formation is referred to as a chief-deputy configuration. The chief is simply the reference point on the chief orbit, and can be either a virtual point in space or an actual spacecraft. In this case study, the chief is the asteroid Apophis, and the deputy is the tracking spacecraft.

Let's assume the only forces acting on the spacecraft are those produced by the gravity field of the Sun, then the relative equations of motion for the spacecraft in formation are given by,

$$
\begin{aligned}
& \ddot{x}=2 \dot{f}\left(\dot{y}-y \frac{\dot{r}_{c}}{r_{c}}\right)+x \dot{f}^{2}+\frac{\mu}{r_{c}^{2}}-\frac{\mu}{r_{d}^{3}}\left(r_{c}+x\right) \\
& \ddot{y}=-2 \dot{f}\left(\dot{x}-x \frac{\dot{r}_{c}}{r_{c}}\right)+y \dot{f}^{2}-\frac{\mu}{r_{d}^{3}} y \\
& \ddot{z}=-\frac{\mu}{r_{d}^{3}} z
\end{aligned}
$$

where $r_{c}$ and $r_{d}=\sqrt{\left(r_{c}+x\right)^{2}+y^{2}+z^{2}}$ are the vectors from the Sun to the formation spacecraft, and to the asteroid respectively, $\mu$ is the gravitational constant of the Sun, $\dot{f}$ is the angular rate of change of the orbit, and $\left\{\begin{array}{lll}x & y & z\end{array}\right\}$ denote the relative rotating coordinate system, called a Hill reference frame measured in the radial $x$, transversal $y$ and normal $z$ directions.

As can be clearly seen, these equations are inherently nonlinear, making it complex to find a closed form set of analytical solutions for the motion of the spacecraft.

An alternate form of representing the orbital motion are Keplerian elements $\left[\begin{array}{llllll}a & e & i & \Omega & \omega & f\end{array}\right]$ where $a$ is the semi-major axis, or a maximum distance from the geometric centre to a point along the orbital path, $e$ is the eccentricity which is a measure of the circularity of the orbit ( $e=0$ being a perfect circle, and $e>1$ being a hyperbola), $i$ is the inclination of the orbital plane, $\Omega$ and $\omega$ are angles determining the location of the line of intersection between the orbital plane and the reference plane, and the periapsis respectively, and lastly $f$ is the true anomaly. The latter term is the only time-varying parameter, and represents the angle between the periapsis of the orbit (the location of the minimum distance from the focal point to the orbit path) and the actual location of the spacecraft at that point in time.

The equations of motion given in (1) can be linearly mapped from Cartesian Hill coordinates to a set of orbital elements by means of a transformation matrix.

$$
\mathbf{X}=\left[A\left(\mathbf{e}_{c}\right)\right] \delta \mathbf{e}
$$

where $\mathbf{X}=\left[\begin{array}{llllll}x & y & z & \dot{x} & \dot{y} & \dot{z}\end{array}\right]^{T}$ is the state vector containing the position, $\mathbf{r}$, and velocity, $\mathbf{v}$, vectors of the spacecraft in the Hill reference frame at a given time, and $\mathbf{e}=\left[\begin{array}{llllll}a & \theta & i & q_{1} & q_{2} & \Omega\end{array}\right]$ is the set of semi-equinoctial orbital elements, where $\theta$ is the true latitude $(\theta=\omega+f)$, and $q_{1}$ and $q_{2}$ are sinusoidal functions based on the eccentricity $e$ and argument of periapsis $\omega\left(q_{1}=e \cos \omega\right.$, $\left.q_{2}=e \sin \omega\right)$. Semi-equinoctial orbital elements were used rather than the classic Keplerian element set in order to avoid singularities that occur in the equations at circular, or near-circular, orbits (i.e. $e=0$ ). The only constraint, or 
assumption required for this linearization is that the semimajor axis of the chief orbit be several orders of magnitude larger than that of the formation orbit.

The subscript ' $c$ ' denotes the chief orbit, while ' $d$ ' denotes that of the smaller deputy, or formation orbit. The difference between the two is given by,

$$
\delta \mathbf{e}=\mathbf{e}_{c}-\mathbf{e}_{d}
$$

The matrix $A$, and the corresponding inverse matrix $A^{-1}$, represent the linear mapping between the Hill frame and the set of orbital elements. The equations for both matrices can be found in [7].

In order to minimize the required station-keeping of the spacecraft, a bounded, or periodic, orbit is advantageous. The bounded orbit is essentially one that, assuming the only forces are those in the standard two-body problem, will return to the same position after a fixed period of time and thus, not need any external control to maintain the spacecraft in the orbit. The conditions of periodicity are such that the period of formation orbit must be equal to the period of the NEO in order not to incur any drift. This is done by setting the difference in semi-major axes equal to zero since the equation for the period of an orbit is a function of only the semi-major axis and gravitation constant, $\mu$.

$$
\delta a=0=\left[\begin{array}{c}
\frac{2 a}{r}\left(2+\frac{3 a}{r} e \cos \theta \cos (2 \omega)\right)+2 \kappa e \sin \theta \\
2 \kappa\left(1+\frac{2 a e}{r} \cos \theta \cos (2 \omega)\right)-\kappa e \sin \theta \\
0 \\
2 a^{2} e \sin \theta \\
\frac{2 a r}{h}\left(1+\frac{2 a}{r} e \cos \theta \cos (2 \omega)+\kappa e \sin \theta\right) \\
0
\end{array}\right] \mathbf{X}(t)(4)
$$

where $h=\sqrt{\mu a\left(1-e^{2}\right)}$ is the angular momentum of the spacecraft, and $\kappa=\frac{a}{r} \frac{\tan \theta}{\cos (2 \omega)}$.

Equation also demonstrates the difference in complexity, and the obvious benefit of switching from the Cartesian Hill frame to an orbital element set.

Given initial conditions for the $x$ and $y$ positions, and the radial velocity $\dot{x}$, it is possible to rearrange (4) to find the required transversal, or along-track, velocity for a periodic orbit. As can be seen from the equation, the calculation is independent of the out-of-plane elements, $z$ and $\dot{z}$.

Once the complete set of initial conditions are determined, the orbit is propagated forward for one complete period. As mentioned earlier, using the Cartesian Hill coordinates this requires the differentiation of 6 time-varying parameters. By switching to an orbital element set, there is only one time- dependant parameter, $f$, that can be calculated over the angular range $[0,2 \pi]$.

A state-transition matrix, $\Phi$, is used to determine the position of the formation spacecraft at any given point in time [3]. The matrix determines the sensitivity of the state vector $\mathbf{X}$ at time $t$ relative to the initial state vector at $t_{0}$.

$$
\begin{aligned}
& {\left[\Phi_{\mathbf{X}}\left(t, t_{0}\right)\right]=\left[\frac{\partial \mathbf{X}(t)}{\partial \mathbf{X}\left(t_{0}\right)}\right]} \\
& =[A(t)]\left[\frac{\partial \boldsymbol{\delta} \mathbf{e}(t)}{\partial \boldsymbol{\delta} \mathbf{e}\left(t_{0}\right)}\right]\left[A^{-1}\left(t_{0}\right)\right]
\end{aligned}
$$

The state vector of the spacecraft can then be determined by,

$$
\mathbf{X}(t) \approx\left[\Phi_{\mathbf{X}}\left(t, t_{0}\right)\right] \cdot \mathbf{X}\left(t_{0}\right)
$$

A periodic orbit can found for almost any point in space surrounding the chief orbit. However, in the case of a tracking mission, there are several mission-specific requirements that restrict the choice of orbit, such as being in close proximity to the surface. As such, an optimizer was used to determine the optimal initial state vector based on a set of objectives and constraints.

\section{Optimal Formation Design Problem}

For the application under investigation, it is required that the tracking spacecraft remains at an almost constant distance from the asteroid. At the same time it is desirable to see the asteroid from different angles. Therefore the problem is to design a periodic orbit that minimizes the difference between the minimum and the maximum distance from the centre of the asteroid:

$$
\min _{\boldsymbol{\rho} \in D} F_{1}=(\max \|\mathbf{r}\|-\min \|\mathbf{r}\|)^{2}
$$

Where the solution vector is defined as:

$$
\boldsymbol{\rho}=\left[\begin{array}{llllll}
x_{0} & y_{0} & z_{0} & \dot{x}_{0} & \dot{z}_{0} & t_{0}
\end{array}\right]^{T}
$$

Another desirable property of the formation would be to bring the plane of the orbit as close to the NEO as possible without crossing into the sphere of influence of the asteroid. This is implemented through the square of the difference between the maximum distance at any point in the formation orbit, and a predetermined minimum distance. In this case, as the spacecraft is to fly as close to the asteroid as possible, the minimum distance is set equal to the sphere of influence of the asteroid, $r_{S O I}$.

$$
\min _{\mathbf{\rho} \in D} F_{2}=\left(r_{S O I}-\max \|\mathbf{r}\|\right)^{2}
$$

However since the spacecraft cannot enter within sphere of influence of the asteroid, an inequality constraint was 
imposed such that the minimum distance between the spacecraft and the NEO is always greater than the radius of the sphere of influence,

$$
C=r_{S O I}-\min \|\mathbf{r}\|<0
$$

\section{OPTIMIZATION PROCEDURE}

The objective functions given in (7) and (9) represent a constrained nonlinear programming problem presenting several local minima in the desired search domain $D$. In particular it is expected that for almost every position on the orbit, i.e. every instant of time, there are several optimal solution vectors that locally minimize the objective function.

A characterization of the search space was performed in order to find not only the global minimum over a range of angular positions along the orbit of the asteroid, but also to find a number of local minima.

We used a behavioral-search algorithm that tries to collect into an archive $A_{g}$ as many feasible local solutions, or proximal to local solutions, as possible. The whole set of feasible local minima is called $X$ in the following. The behavioral search is hybridized with a domain decomposition technique in order to extend the exploration of the search space.

\section{A. Behavioral-Based Search}

A population of virtual agents is deployed in the search space. Each agent is endowed with a set of basic actions forming a behavior. The entire population evolves through a number of steps toward the feasible set $X$. At each evolutionary step, the agents collect clues about the environment and implement actions according to an action selection mechanism. Some of the actions are devoted to acquire new information, others to displace the agents toward local minima, other actions are instead made to exchange information among the agents.

The basic idea is that most of the bio-inspired global optimization approaches implement some form of basic behavior derived from nature: from mating of Evolutionary Algorithms (EA) to the flying of bird flocks in Particle Swarm Optimization (PSO) to the social behavior of ants in Ant Colony Optimization (ACO).

Some of those behaviors can be classified as social, such as crossover in genetic algorithms, because they are devised to exchange pieces of information among the individuals. Other behaviors can be classified as individualistic because they aim at improving the individual status of each individual. We implement a set of actions derived from PSO, EA and DE (Differential Evolution) and a very simple, basic action selection mechanism for individualistic behavior (see Fig. 3). Each agent can perform repeatedly three types of actions until an improvement is registered or a maximum number of attempts is reached.

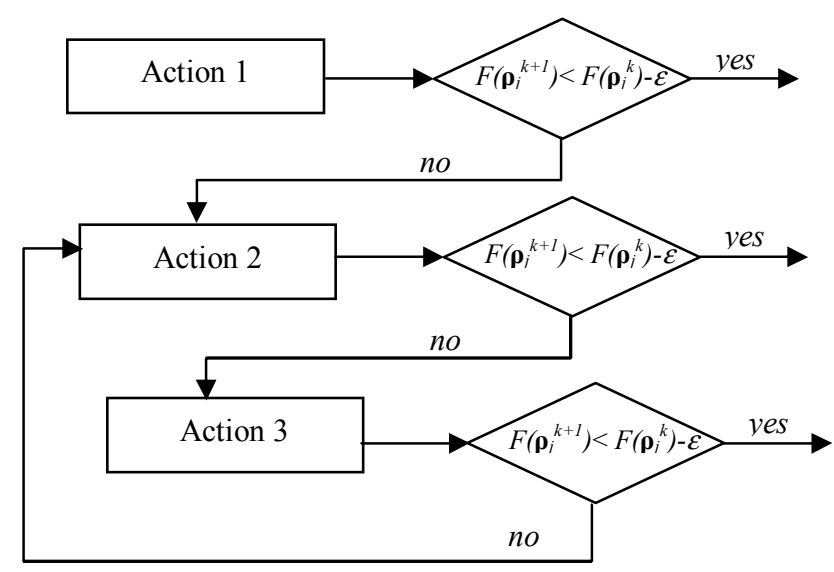

Fig. 3. Action selection mechanism

After an initialization is performed by sampling the solution space by Latin Hypercube, each solution vector is associated to an agent. Each agent is then evaluated and social behaviors (such as crossover) are implemented. After exchanging information with the other agents, each one can implement a set of individualistic behaviors (such as mutation, for example). The archive collects all the solutions that are considered locally optimal at every evolutionary step. Furthermore, at termination the entire population is added to the archive. The overall algorithm, called Multiagent Collaborative Search (MACS), is represented in Fig. 4.

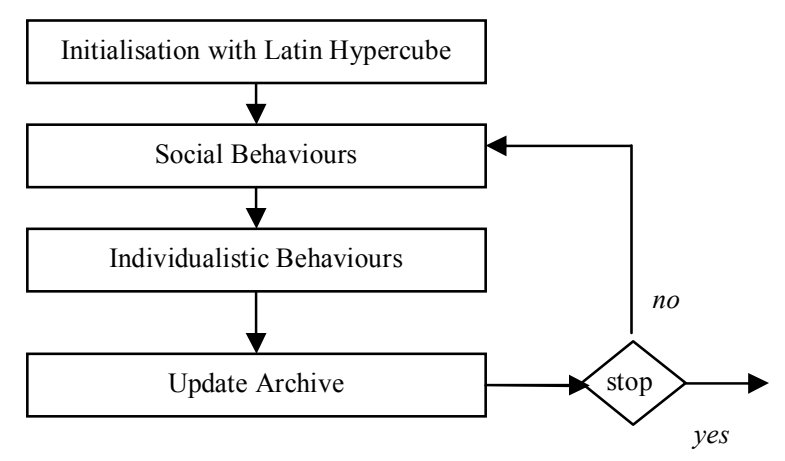

Fig. 4. Overall algorithm for the behavioral-bases search.

The search is terminated after a given number of function evaluations. For further details on the behavioral search the interested reader can refer to [9]-[12].

\section{B. Domain Decomposition}

Since the number of local minima is expected to be finite (for physical reasons), the search space can be decomposed into a finite number of subsets, each containing a portion of the optimal set $X$ such that $X \cap D \neq 0$. The initial domain $D$ is progressively decomposed into smaller domains $D_{l} \subseteq D$ according to a decomposition strategy. The decomposition strategy is based on the output of the stochastic search step and produces a number $M$ of subdomains $D_{l}$ such that: 


$$
\bigcup_{l=1}^{M} D_{l}=D
$$

Decomposition (11) is then iteratively applied to the subdomains $D_{l}$ that need further exploration, so that:

$$
\bigcup_{l=1}^{M} D_{l}^{(d)}=D_{l}^{(d-1)}
$$

where $d$ is the decomposition depth.

In this implementation we use a simple regular cutting of each coordinate into two halves. Since the interest is to widely explore the search space and to collect as many elements of $X$ as possible, after each stochastic search the subdomains $D_{l}$ with the least number of collected samples belonging to $X$ is selected for further decomposition provided that the number of times $n_{b}$ its parent subdomains have been already decomposed without improvement is below a given threshold.

We use the following merit function to balance the search of completely unexplored subdomains against those containing elements of $X$ :

$$
\psi_{D_{l}}=(1-v) \varpi_{D_{l}}+v \varphi_{D_{l}}
$$

where $\varpi_{D_{l}}$ is the density of already discovered solutions in $D_{l}, \varphi_{D_{l}}$ is the best fitness in $D_{l}$ and $v$ is a weighting factor used to favor either convergence or exploration.

The partitioning cycle is stopped when a given number of subdomains have been generated, or when the size of the archive has been exceeded.

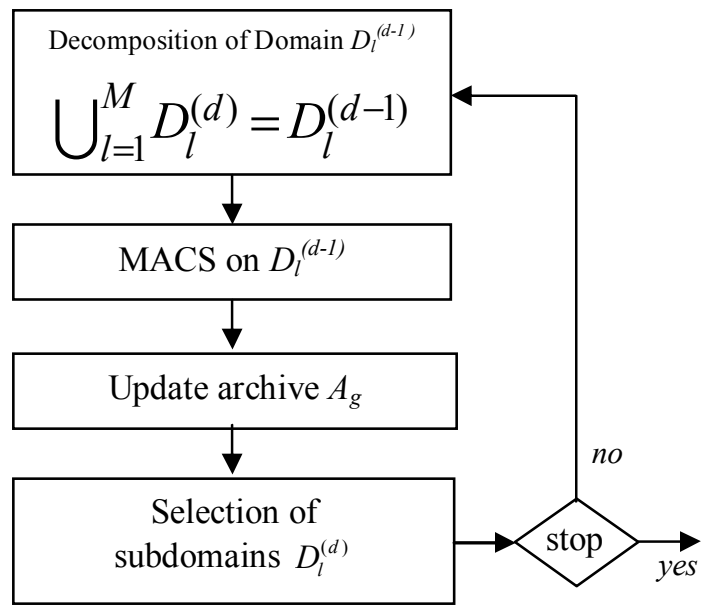

Fig. 5. Hybrid domain decomposition and stochastic search.

The overall algorithm combining the MACS and the domain decomposition technique is called EPIC in the following (Evolutionary Programming and Interval Computation) [10]-[12].

\section{Constraint Handling Technique}

The algorithm described above solves bound-constrained problems. Since in most of the cases however, constraints are nonlinear, an extension of the algorithm has been developed in order to take into account nonlinear inequality constraints.

At each generation, the population of agents is divided into two subpopulations and a different objective function is assigned to each one, namely one subpopulation aims at minimizing the original objective function while the other aims at minimizing the residual on the constraints, defined as,

$$
\min _{\mathbf{p} \in D} F=\sum_{j=1}^{m} \max \left(\left[0, C_{j}\right]\right)
$$

The two subpopulations are evolved in parallel and agents are allowed to jump from one population to the other, i.e. if a feasible agent becomes infeasible it is inserted in the subpopulation of infeasible agents and assigned to the solution of the constraints, on the other hand if an infeasible agent becomes feasible it is inserted in the population of feasible individuals and allocated to the minimization of the original bound constrained objective function $F$. As a result, the final optimal solution is either feasible or minimizes infeasibilities. This procedure does not maintain feasibility for any agent, therefore once a feasible set has been found the perception mechanism is used to ensure that every move maintains the feasible population inside the feasible set. If $F^{*}$ is the value of the objective function of an agent $\boldsymbol{\rho}$ inside the feasible set, the objective function of a new agent generated from $\boldsymbol{\rho}$ is then augmented with the maximum among the residuals $R$ on the constraints:

$$
\min _{\mathbf{p} \in D} F=\left\{\begin{array}{l}
F^{*} \text { if every } C_{j} \leq 0 \\
F^{*}+\max (C) \text { if any } C_{j}>0
\end{array}\right.
$$

The described strategy co-evolves two populations with two different goals, allows a flexible search for feasible optimal solutions: in fact through the described use of the perception mechanism, feasibility can be enforced on all feasible solutions. In this case the exploration of the solution space may be over penalized, thereby reducing the convergence rate. Therefore in order to search more extensively along the boundary of the feasible region, a subset of the feasible solutions is allowed to temporary violate the constraint while preserving the feasibility of at least the best solution.

\section{Definition of the Search Space}

A number of boundary conditions were set to restrict the search space and improve the speed of the program. Given that the objective functions are designed to find orbits in close proximity to the NEO, small limits were set for the distance between the spacecraft and asteroid.

$$
\{x, \quad y, \quad z\} \in\left[\begin{array}{ll}
-5 & 5
\end{array}\right] \mathrm{km}
$$


Most matrix functions, including the optimizers in question, work better when the search space values are around unity. Given that the velocities are quite small, on the order of $10^{-9} \mathrm{~km} / \mathrm{s}$, the input parameters were scaled up by a factor of $10^{9}$.

$$
\left\{\begin{array}{ll}
\dot{x}^{*} & \dot{z} *
\end{array} \in\left[\begin{array}{ll}
-100 & 100
\end{array}\right] \mu \mathrm{m} / \mathrm{s}\right.
$$

Lastly the time duration was set for 30 days starting on 01 January 2010. The time is measured in modified Julian Days from 01 Jan 2000 12:00:00 GMT.

$$
t \in\left[\begin{array}{ll}
3693.5 & 3723.5
\end{array}\right] \text { MJD2000 }
$$

\section{RESULTS}

\section{A. Optimized Solution Vector}

EPIC was run with a total of 10 agents exploring the search space. The number of subdomains for the first run was limited to 5 and the maximum number of function evaluations per subdomain was set to 10000 . Fig. 6 shows the distribution of the minima at the end of the search. Some regions are clearly identifiable.

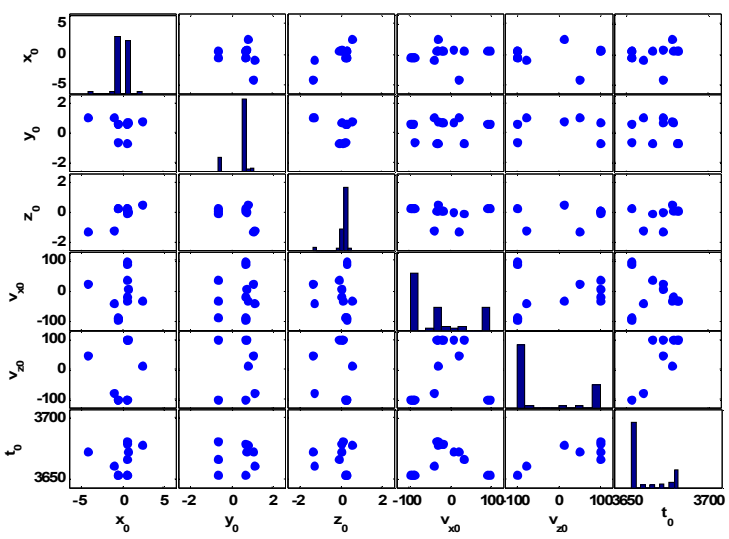

Fig. 6. The cross-comparison of the solution vector $\boldsymbol{\rho}$ using EPIC. The distances are measured in $\mathrm{km}$, the velocities in $10^{-9} \mathrm{~km} / \mathrm{s}$ and time in MJD2000.

From this search, it is simple to find the smallest objective function value and extract the optimal initial state vector for the orbit, and starting date.

Fig. 7 shows the resulting formation orbit. The optimized initial state vector is,

$\left(\mathbf{X}_{0}\right)_{\text {opt }}=\left[\begin{array}{c}x_{0}=575.44 \\ y_{0}=646.62 \\ z_{0}=304.15 \\ \dot{x}_{0}=9.738 e^{-5} \\ \dot{y}_{0}=-3.517 e^{-4} \\ \dot{z}_{0}=-1.00 e^{-4}\end{array}\right] \Leftrightarrow(\delta \mathbf{e})_{\text {opt }}=\left[\begin{array}{c}\delta a=0 \\ \delta e=-0.0884 \\ \delta i=1.564 \\ \delta \Omega=-2.347 \\ \delta \omega=-23.62 \\ \delta f_{0}=0.468\end{array}\right] \times 10^{-7}$

$t_{0}=3653.0$ shown in both Hill coordinates, and Keplerian orbital elements, where the units are meters, seconds, degrees and MJD2000.

It is interesting to note that both values in the objective function only differ after the tenth significant digit. A byproduct of the equations of motion is that the closer the orbit is to the asteroid, the more normal the orbital plane becomes relative to the asteroid velocity vector. Conversely, the farther away the formation orbit is from the chief point, the larger the semi-major axis and the more parallel the orbital plane.

Fig. 7 shows the formation orbit relative to the asteroid. The period of the formation orbit is equal to the time it takes for the asteroid to make one complete revolution about the Sun.

The final orbit has a slight deviation from the desired orbital plane, on the order of $300 \mathrm{~km}$. This orbit provides a relatively consistent distance from the surface of the asteroid and provides good coverage of the surface.
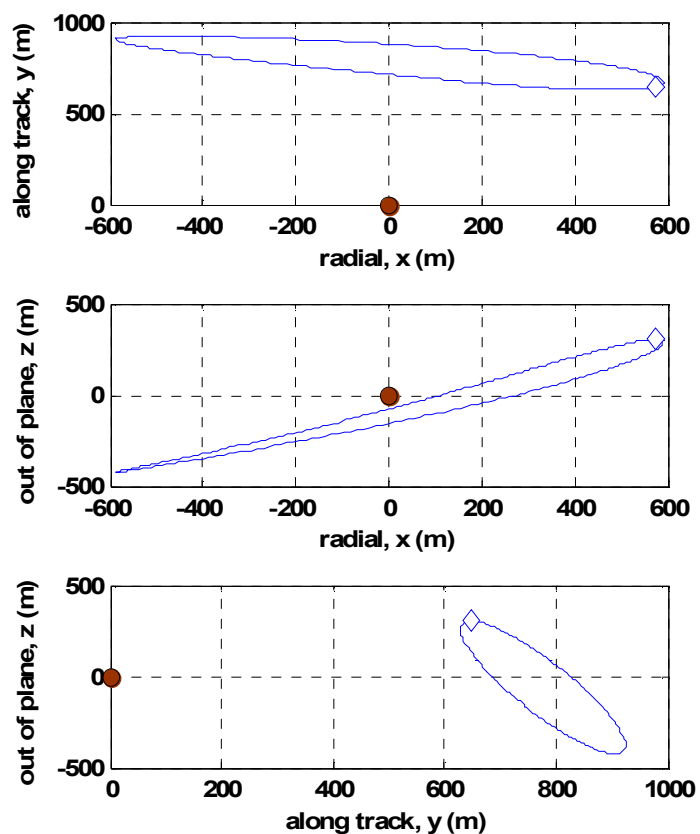

Fig. 7. Orthographic view of the optimal formation orbit, including the initial state vector (shown as a diamond), and location relative to Apophis (shown as a brown circle).

Note from Fig. 6, that along the $t_{0}$ coordinate the distribution is almost continuous. This evidence confirms the fact that an optimal configuration exists for every position along the orbit. It also suggests that a globally optimal position along the orbit exists that give the minimum of both objective functions.

A second optimization was conducted extending the range of $t_{0}$ to 1 Earth year, from 01 January - 31 December 2010. The period of Apophis is slightly less than that of Earth; 323.6 days. By searching for a full year, we can see the effect the orbital position of the asteroid on the initial state 
vector for formation orbit.

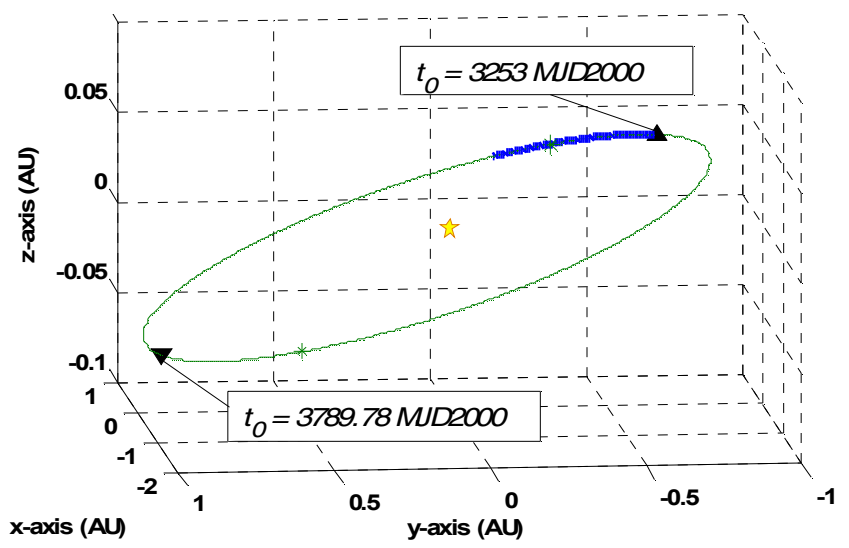

Fig. 8. Orbit of Apophis showing the optimal $t_{0}$ for the two solutions (shown as black triangles). The apoapsis and periapsis are also shown for reference (green stars) with the first solution near the periapsis, and the second solution near the apoapsis. The first search space for $t_{0}$ is shown in blue (bold).

Table 2 lists the upper and lower bounds on the solution vector used in the second optimization process.

TABLE 2

UPPER AND LOWER BOUNDS ON THE SEARCH SPACE FOR EACH VARIABLE IN THE SOLUTION VECTOR FOR THE SECOND OPTIMIZATION

\begin{tabular}{ccccccc}
\hline \hline & $\begin{array}{c}x \\
(\mathrm{~km})\end{array}$ & $\begin{array}{c}y \\
(\mathrm{~km})\end{array}$ & $\begin{array}{c}\dot{x} * \\
(\mathrm{~km})\end{array}$ & $\begin{array}{c}\dot{z} * \\
\left(10^{-9}\right. \\
\mathrm{km} / \mathrm{s})\end{array}$ & $\begin{array}{c}t^{*} \\
\left(10^{-9}\right. \\
\mathrm{km} / \mathrm{s})\end{array}$ & $\begin{array}{c}t_{0} \\
(\mathrm{MJD}\end{array}$ \\
\hline $\begin{array}{l}\text { Lower } \\
\text { bound }\end{array}$ & -5 & -5 & -5 & -1000 & 100 & 3652 \\
\hline $\begin{array}{l}\text { Upper } \\
\text { bound }\end{array}$ & 5 & 5 & 5 & 100 & 100 & 4018 \\
\hline \hline
\end{tabular}

The optimal initial state vector is given below, and the resulting formation orbit in Fig. 9.

$$
\begin{aligned}
& \left(\mathbf{X}_{0}\right)_{o p t}=\left[\begin{array}{c}
x_{0}=-529.88 \\
y_{0}=-664.10 \\
z_{0}=468.73 \\
\dot{x}_{0}=-1.00 e^{-4} \\
\dot{y}_{0}=1.95 e^{-4} \\
\dot{z}_{0}=-9.99 e^{-5}
\end{array}\right] \Leftrightarrow(\delta \mathbf{e})_{o p t}=\left[\begin{array}{c}
\delta a=0 \\
\delta e=0.038 \\
\delta i=-1.701 \\
\delta \Omega=8.389 \\
\delta \omega=-40.92 \\
\delta f_{0}=-0.755
\end{array}\right] \times 10^{-7} \\
& t_{0}=3789.76
\end{aligned}
$$

As with the previous state vector, the units are meters, seconds, degrees and MJD2000.

\section{CONCLUSION}

This paper presents the design and methodology for finding an optimal orbit for a spacecraft flying in formation with an asteroid. The final orbit presented meets the requirements for a NEO tracking mission: close proximity to the asteroid with minimal variation in distance in order to minimize the pointing errors of the measurement devices.
The development of a periodic orbit also meets the general requirement of cost savings in both propulsion and mass.

These orbits were optimized for a NEO tracking mission, however the methodology can be extended to any mission based on the primary and secondary mission requirements.
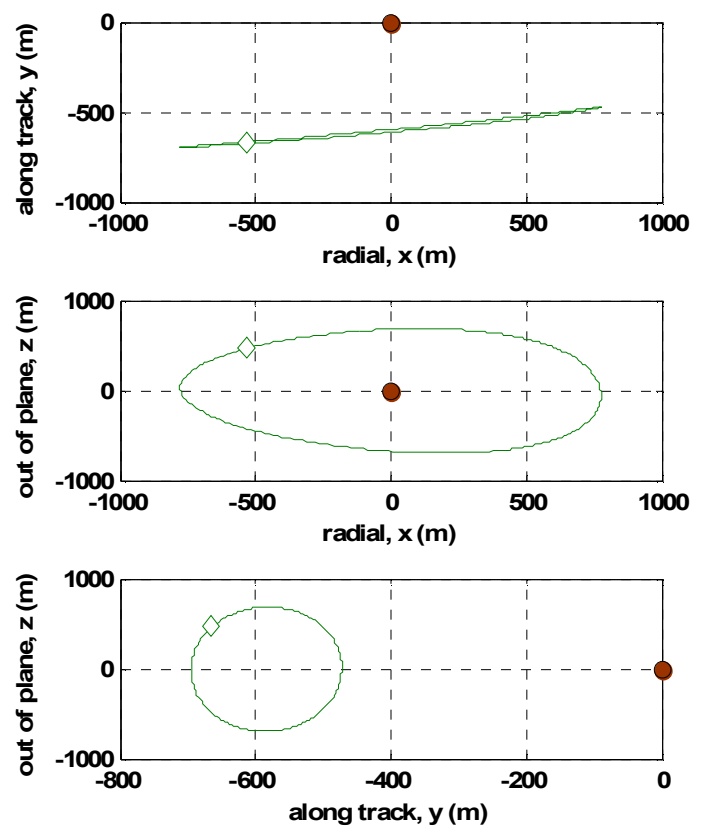

Fig. 9. Orthographic view of the formation orbit optimized over an 1 year period, showing the initial state vector (shown as a diamond), and location relative to Apophis (shown as a brown circle).

\section{REFERENCES}

[1] Space Mission Priorities for Near-Earth Object Risk Assessment and Reduction, Near-Earth Object Mission Advisory Panel, ESA Report, 2004.

[2] Recommendations on the detection of asteroids and comets potentially dangerous to humankind, Council of Europe Resolution 1080, 1996.

[3] Final Report from the Workshop on NEOs: Risks, Policies and Actions, Global Science Forum, Organisation for Economic Cooperation and Development, 2003.

[4] 99942 Apophis (2004 MN4) Impact Risk (online resource), NASA Near Earth Object Program http://neo.jpl.nasa.gov/risk/a99942.html (link valid as of 2007.03.23).

[5] C. Maddock, J. P. Sanchez Cuartielles, M. Vasile and G. Radice, "Comparison of Single and Multi-Spacecraft Configurations for NEA Deflection by Solar Sublimation", New Trends in Astrodynamics and Applications III, American Institute of Physics Conference Proceedings, pp. 303-316, 2007.

[6] R. Battin, An Introduction to the Mathematics and Methods of Astrodynamics, Revised Edition. AIAA Education Series, Virginia, 1999.

[7] H. Schaub and J. L. Junkins, Analytical Mechanics of Space Systems, AIAA Education Series, Virginia, 2003.

[8] D.-W. Gim and K. T. Alfriend, "The State Transition Matrix for Relative Motion of Formation Flying Satellites", Advances in the Astronautical Sciences, vol. 112, pp. 1021-1042, 2002.

[9] M. Vasile and M. Locatelli, "A Behavioural-Based Approach to the Global Optimisation of Space Mission Design Problems", EURO XXI, Reykjavik, Iceland, July 2006.

[10] M. Vasile, "A Hybrid Multi-Agent Collaborative Search Applied to the Solution of Space Mission Design Problems", Global Optimization Workshop, Almeria, Spain, 18-22 September 2005. 
[11] M. Vasile, "A Behavioral-based Meta-heuristic for Robust Global Trajectory Optimization", IEEE Congress on Evolutionary Computing 2007, Singapore, 25-28 September 2007.

[12] Vasile M., Robust Mission Design Through Evidence Theory and Multiagent Collaborative Search. Annals of the New York Academy of Sciences, Vol 1065: pp. 152-173, December 2005. 